\title{
Relative value of oyster reef as habitat for estuarine nekton in Galveston Bay, Texas
}

\author{
Gregory W. Stunz ${ }^{1, *}$, Thomas J. Minello ${ }^{2}$, Lawrence P. Rozas ${ }^{3}$ \\ ${ }^{1}$ Harte Research Institute for Gulf of Mexico Studies and Department of Life Sciences, Texas A\&M University-Corpus Christi, \\ 6300 Ocean Drive, Corpus Christi, Texas 78412, USA \\ ${ }^{2}$ SEFSC, Galveston Laboratory, NOAA Fisheries Service, 4700 Avenue U, Galveston, Texas 77551, USA \\ ${ }^{3}$ SEFC/Estuarine Habitats and Coastal Fisheries Center, NOAA Fisheries Service, 646 Cajundome Blvd., Lafayette, \\ Louisiana 70506, USA
}

\begin{abstract}
Biogenic reefs formed by dense aggregations of the eastern oyster Crassostrea virginica are a dominant feature in most estuarine systems along the Atlantic and Gulf of Mexico. Oyster reefs are complex in their structural nature and have long been recognized for their potential habitat value. However, relatively few studies have characterized nekton abundance in this complex habitat type, and live high-relief oyster beds have been particularly difficult to sample with conventional gear. We used a quantitative sampling device to compare nekton use among high-relief live oyster reef, vegetated marsh edge Spartina alterniflora, and nonvegetated bottom habitat types. During $1 \mathrm{yr}$ of seasonal sampling we collected 3791 fishes and 12386 crustaceans representing 38 and 21 different species, respectively. Density and biomass of most fishes and crustaceans were significantly higher in oyster reef than over nonvegetated bottom. For benthic crustaceans, oyster reef supported a higher density and biomass than vegetated marsh edge. Nektonic crustaceans were generally more abundant in marsh edge than on oyster reef. Species composition and richness varied among habitat types and season; however, richness was highest in oyster reef, followed by marsh edge, and lowest on nonvegetated bottom, except during seasonal low densities during winter. Species composition and size differences were observed among habitat types. Our results show that oyster reef supports a high density, biomass, and richness of estuarine nekton in relation to typically examined estuarine habitat types and has the potential to be an essential habitat. Identifying and quantifying the role of oyster reefs will be critical to implementing effective management for essential fish habitat.
\end{abstract}

KEY WORDS: Oyster reef $\cdot$ Essential fish habitat $\cdot$ Nekton habitat use $\cdot$ Estuarine habitat

\section{INTRODUCTION}

The ability of estuaries to support high productivity and abundance of marine life clearly stems from the availability of habitat and ecological services these areas provide. High nekton densities in shallow estuarine habitats and the value of these habitats for survival and growth have by been well-documented by numerous investigators (e.g. Weinstein 1979, Heck \& Thoman 1981, Boesch \& Turner 1984, Kneib 1984, Day et al. 1989, Baltz et al. 1993, Beck et al. 2001, Minello et al. 2003). Densities of fish and crustaceans often vary among putative habitat types, and characterizing density patterns provides useful information on their relative value (Baltz et al. 1993, Minello 1999). Common estuarine habitat types assessed typically include seagrass beds, intertidal marshes, and both intertidal and subtidal mud flats (Day et al. 1989). However, few studies have characterized relative nekton abundance on oyster reef compared to other common estuarine habitat types, principally due to the difficulties in using conventional gear for quantitatively sampling live, complex, high-relief oyster reef (but see Zimmerman et al. 1989, Wenner et al. 1996, Coen et al. 1999, 
Minello 1999, Stunz et al. 2002a, Peterson et al. 2003, Coen et al. 2007). Thus, most work on estuarine nekton habitat use has contrasted nonvegetated bottom with vegetated areas such as subtidal seagrass meadows (see reviews by Orth et al. 1984, Pollard 1984, Minello 1999) or salt marshes (Zimmerman et al. 1984, Baltz et al. 1993, Rozas \& Zimmerman 2000).

The complexity that makes oyster reef difficult to sample also may be the characteristic making this a valuable estuarine habitat type. The typical 3-dimensional structure provided by a live oyster complex has often been compared to that in coral reefs (Harding \& Mann 1999, Tolley \& Volety 2005). For example, this complexity can provide a spawning substrate for a variety of nekton (Runyan 1961, Breitburg et al. 1999) or a refuge from predation. Several experimental studies have documented that nekton preferentially select oyster reef over other common estuarine habitat types (Posey et al. 1999, Stunz et al. 2001), often resulting in lower mortality rates (Stunz et al. 2002a, Grabowski 2004) or influencing benthic trophic cascades (Grabowski et al. 2008). Additionally, reefs provide a food source for a variety of marine life (Harding \& Mann 2001, Grabowski 2004) leading to rapid growth and increased survivorship (Sogard 1997, Stunz et al. 2002b, Shervette \& Gelwick 2007). However, it is still unclear whether oyster reefs actually support high densities of nekton relative to other potential habitat types.

A variety of approaches have been used with different gear in attempts to quantify nekton use of oyster reef over the last 2 decades (see review by Coen \& Grizzle 2007). Many studies have successfully collected nekton on oyster reef but have focused only on particular species, in part because of sampling constraints from gear limitations (Micheli \& Peterson 1999, Meyer \& Townsend 2000). For example, gill nets, suction dredges, traps, SCUBA, and trawls (Mann \& Harding 1997, Lenihan et al. 1998, Mann \& Harding 1998, Harding \& Mann 2001, Lenihan et al. 2001, Grabowski et al. 2005) have all been used with some caveats regarding gear biases (Rozas \& Minello 1997). Alternatively, others have used proxies to represent oyster reef and sampled trays of oysters (Lehnert \& Allen 2002, Plunket \& La Peyre 2005, Rodney \& Paynter 2006), unconsolidated oyster shell (Stunz et al. 2002a, Shervette \& Gelwick 2008), or the nonvegetated bottom adjacent to a reef (Glancy et al. 2003). Perhaps the most successful quantification of nekton over oyster reef has been achieved using intertidal lift nets (Wenner et al. 1996, Tolley \& Volety 2005). Lift nets that depend upon a receding tide are effective in areas with predictable tides, but are ineffective in many areas such as subtidal reefs or where tides are unpredictable because meteorological events dominate astronomical tides, e.g. the
Gulf Coast of the United States (Rozas 1995). Zimmerman et al. (1989) used a drop sampler on oyster reef in Galveston Bay, Texas, but only during winter and summer, and the efficiency of the gear was not assessed. Thus, making accurate and comparative density estimates of the nekton community on oyster reef in relation to other estuarine habitat types will continue to be problematic until a gear is developed that is effective both in oyster reef and other major habitat types.

A lack of data on nekton using oyster reef, especially in relation to other estuarine habitat types, has prevented estuarine ecologists and resource managers from developing a better understanding of the habitat role oyster reefs play in estuarine systems. Thus, the goal of the present study was to simultaneously compare nekton habitat value among high-relief live oyster reefs, vegetated marsh edge, and nonvegetated bottom. Our approach included the construction of multiple experimental oyster reef plots, and we used a highly efficient drop sampler to compare nekton densities on these plots with adjacent putative habitat types seasonally over 1 yr.

\section{MATERIALS AND METHODS}

Study site and design. The present study was conducted in West Galveston Bay on the upper Texas coast, USA (Fig. 1). West Bay is a barrier-built estuary that is mesohaline to euhaline with 2 major inlets (Bolivar Roads and San Luis Pass) that connect the estuary to the Gulf of Mexico. The tides are mixed and primarily diurnal with a mean daily range of $0.3 \mathrm{~m}$ (Galveston Pier 21, National Ocean Service, National Oceanic and Atmospheric Administration [NOAA]). Spartina alterniflora is the dominant intertidal emergent marsh and remains flooded about $78 \%$ of the year, with higher flooding durations during the fall and spring (Minello \& Webb 1997). Other major habitat types include oyster reefs Crassostrea virginica, which cover 10800 ha, or about $10 \%$, of the Galveston Bay bottom (Powell et al. 1995), and sandy or muddy nonvegetated bottom. Our study area at Gang's Bayou on Galveston Island is a location where fragmented salt marsh, oyster reefs, and shallow open water are all in close proximity (Fig. 1). Our sample sites included marsh edge (ME), oyster reef (OYS) plots, and shallow nonvegetated bottom (SNB) in open water.

During an exceptionally low tide in January 2000, when an entire reef complex was exposed, we created 50 replicate OYS plots in the study area. Each reef plot consisted of 571 of live oysters spread over a circular area $0.8 \mathrm{~m}$ in diameter. The plots were created by moving live oysters from the reef to create a patch $(\sim 20$ to $30 \mathrm{~cm}$ high) over adjacent bare sand bottom within the 

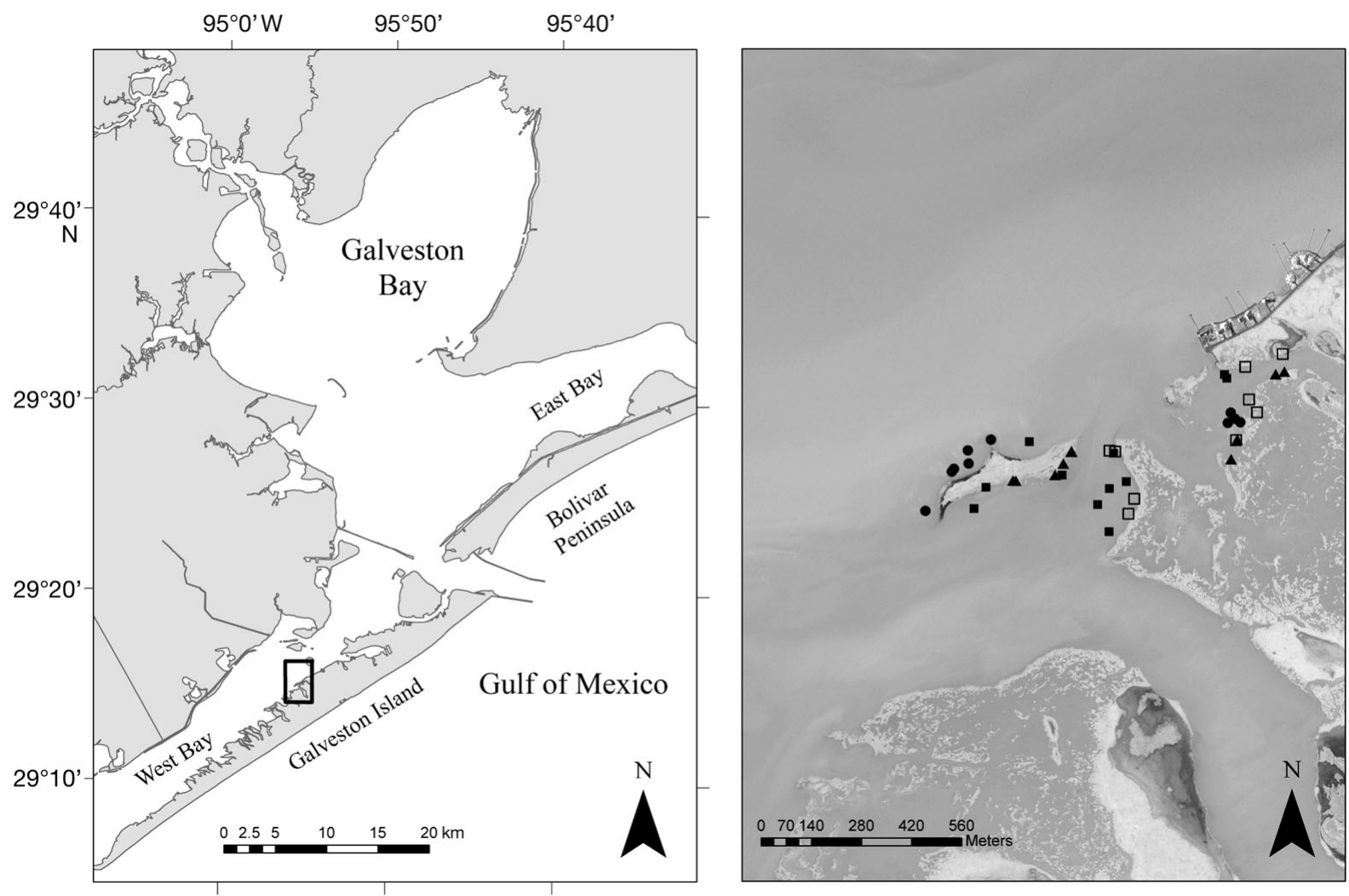

Fig. 1. General sampling locations (November 2000 only) and spatial arrangment at Gang's Bayou in West Galveston Bay, Texas,

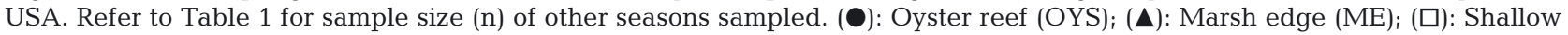
nonvegetated bottom $<10 \mathrm{~m}$ from the marsh shoreline (SNB Near); and ( $\mathbf{\square})$ : Shallow nonvegetated bottom $>10 \mathrm{~m}$ from the marsh shoreline (SNB Far)

overall OYS complex. The center of each plot was marked using a stake visible above the water line. The reef plots were left undisturbed for a minimum of 5 mo before sampling. We also selected corresponding ME sites and SNB areas (Fig. 1). The ME interface is the ecotonal zone between open water and the emergent vegetation, Spartina alterniflora (Zimmerman et al. 1984, Baltz et al. 1993), and our ME samples were in the vegetation within $\sim 1 \mathrm{~m}$ of the edge. Our SNB sites were further subdivided into SNB Near $(<10 \mathrm{~m}$ from $\mathrm{ME})$ and SNB Far ( $\geq 10 \mathrm{~m}$ from ME); all SNB sites were $>10 \mathrm{~m}$ from OYS.

In each of 4 seasons during 2000-2001 (spring: May 15-16, 2000; summer: August 14-15, 2000; fall: November 6-7, 2000; and winter: February 12-13, 2001), we used $2.6 \mathrm{~m}^{2}$ drop enclosure samplers (Zimmerman et al. 1984) to estimate densities of nekton on 4 habitat types; OYS, ME, and SNB (both SNB Near and SNB Far). Our objective was to collect 10 samples from each habitat type during each season, but low water prevented the collection of some ME samples during winter (Table 1). Specific oyster plots were selected randomly for sampling each season, and other habitat types were chosen using random numbers and a grid placed over an aerial photograph. No plot or other site was sampled more than once during the study. Two boats and crews of at least 3 persons each were used simultaneously to collect these samples. The order was occasionally modified to avoid disturbing nearby sampling locations.

To collect samples, the cylindrical sampler was suspended from a boom mounted on a shallow draft boat. We pushed the boat from the stern to minimize disturbance and dropped the cylinder rapidly to enclose the

Table 1. Habitat types examined in this study and the sample size (n) by season. OYS: oyster reef mounds; ME: marsh edge emergent vegetation within $1 \mathrm{~m}$ of the shoreline; SNB Near: shallow nonvegetated bottom $<10 \mathrm{~m}$ from the marsh shoreline; SNB Far: Shallow nonvegetated bottom $>10 \mathrm{~m}$ from the marsh shoreline

\begin{tabular}{|c|c|c|c|c|c|}
\hline \multirow[t]{2}{*}{ Habitat category } & \multicolumn{5}{|c|}{ - Sample size $(\mathrm{n})$} \\
\hline & Spring & Summer & Fall & Winter & Total \\
\hline OYS & 10 & 10 & 10 & 10 & 40 \\
\hline $\mathrm{ME}$ & 10 & 11 & 10 & 6 & 37 \\
\hline SNB Near & 9 & 10 & 10 & 10 & 39 \\
\hline SNB Far & 12 & 9 & 10 & 11 & 42 \\
\hline Total & 41 & 40 & 40 & 37 & 158 \\
\hline
\end{tabular}


sampling area. Immediately after deployment, we measured water temperature, dissolved oxygen, salinity, and turbidity as described by Minello \& Zimmerman (1992). Water depth was determined using a meter stick at each sample site and averaging 5 measurements; depth was measured from the surrounding sediment and not from the top of the OYS. For the SNB samples, we measured the distance from the center of the sampler to the nearest ME. As necessary at ME sites, stems of emergent vegetation were clipped at the ground level to facilitate collection of animals. To efficiently remove animals from the oyster plots, all oysters were removed after the sampler enclosed the plot, and this material was carefully washed and searched for crabs and cryptic fishes hiding within the oyster clumps. The enclosed area was then swept with dip nets and drained by pumping water out through a $1 \mathrm{~mm}$ mesh net. Any remaining animals were removed by hand. Samples were stored in formalin for identification and enumeration in the laboratory, where they were separated from detritus, shell hash, and plant parts and identified to the lowest feasible taxon. We used the nomenclature of Perez Farfante \& Kensley (1989) for penaeid shrimps and identified species using the protocol described in Rozas \& Minello (1998). Total length (TL) of fishes and shrimps and carapace width of crabs were measured to the nearest mm. If more than 20 individuals were caught for each species or group, the largest and smallest and 20 other random individuals were measured. Individuals of a species in each sample were pooled to determine biomass (wet wt) to the nearest $0.1 \mathrm{~g}$. Species richness was defined as the number of species identified in a sample.

Catch efficiency study. Drop samplers efficiently sample shallow marsh habitat and have been used previously to sample nekton in a variety of estuarine habitats (Rozas \& Minello 1997), including oyster reef (Zimmerman et al. 1989). The efficacy of this gear for sampling oyster reef, however, has not been demonstrated. Therefore, we tested the efficiency of recovering nekton from the drop sampler during May 2001 using extra OYS plots. In each of 10 drop samples on oyster reef, we stocked brown shrimp Farfantepenaeus aztecus, spot Leiostomus xanthurus, killifish Cyprinodon variegates, and pinfish Lagodon rhomboides (10 individuals of each species) that had been marked by fin clips. After nekton were held in the sampler for approximately $1 \mathrm{hr}$, the enclosure was sampled as described above. We calculated the mean percent recovery for each species.
Statistical design. We tested for differences in fish and decapod crustacean densities and biomass among habitat types with ANOVA using the general linear model procedure in SAS $9.1(\alpha=0.05)$. To assess catch efficiency, we used a 1-way ANOVA to assess differences in recovery rates among the 4 species. For each season, we used a 1-way ANOVA to test the main effect of habitat type (OYS, ME, SNB Near, and SNB Far) for environmental data and abundant nekton. Although we set our significance level at 0.05 , we also calculated adjusted $\alpha$ levels for the habitat effect for each species using the sequential Bonferroni method described by Rice (1989) to buffer against error from testing a hypothesis on several species or environmental variables. We used a priori contrasts to test for significant differences in nekton densities and environmental variables between OYS and ME, OYS and SNB, and SNB Near and SNB Far (for an example, see Table 2). Before analyses, all observations were converted to number of nekton $\mathrm{m}^{-2}$, and a $\log _{10}(x+1)$ transformation was used on these density data to ensure homogeneity of variance and normality of the residuals.

We tested for differences in density among habitats for selected economically and ecologically important species during their peak recruitment season. We calculated mean densities and sizes for red drum Sciaenops ocellatus from November 2000 samples (Holt et al. 1983); pinfish from May 2000 and February 2001 samples (Levin et al. 1997, Pattillo et al. 1997); blue crab from all sampling dates (Pile et al. 1996, Blackmon \& Eggleston 2001); brown shrimp from May, August, and November 2000 samples; and white shrimp Litopenaeus setiferus from August and November 2000 samples (Pattillo et al. 1997). A small number of penaeid shrimp ( 130 total) were damaged during the collection process, and only carapace length (CL) could be measured. We estimated TL for these speci-

Table 2. Analysis of variance table for comparing 4 habitat types including OYS, ME, and SNB Near and SNB Far (see Table 1 for abbreviations). The model tests for the main effect of habitat type, and a priori contrasts compare specific habitat types. The dependent variable used in this example is the $\log _{10}(x+1)$ transformation of total macrofauna density (sum of total fishes and crustaceans) for May samples

\begin{tabular}{|lrrrrr|}
\hline Source & df & SS & MS & Fvalue & p \\
\hline Habitat type & 3 & 14.665 & 4.888 & 31.561 & $<0.001$ \\
$\quad$ Contrasts & & & & & \\
$\quad$ OYS vs. ME & 1 & 3.025 & 3.025 & $19.531<0.001$ \\
$\quad$ OYS vs. SNB Near + SNB Far & 1 & 14.054 & 14.054 & 90.741 & $<0.001$ \\
$\quad$ SNB Near vs. SNB Far & 1 & 0.053 & 0.053 & 0.341 & 0.563 \\
Residual error & 37 & 5.731 & 0.155 & & \\
\hline
\end{tabular}


mens using conversion equations (white shrimp: 2 to $18 \mathrm{~mm} \mathrm{CL}$, TL $=-0.53+5.06 \times \mathrm{CL}, \mathrm{R}^{2}=0.99$; brown shrimp: 1.8 to $18 \mathrm{~mm} \mathrm{CL}, \mathrm{TL}=0.28+4.5 \times \mathrm{CL}, \mathrm{R}^{2}=$ 0.98). A 1-way ANOVA (with a seasonal blocking variable where appropriate) was used to compare species mean densities, biomass, and sizes by habitat type during their peak recruitment. Mean differences among habitats for each species were further tested using Tukey's HSD ( $\alpha=0.05$; Day \& Quinn 1989). For this analysis we compared only 3 habitats: OYS, ME, and SNB (SNB Near and SNB Far combined). Finally, we assessed differences in species richness by habitat type seasonally using a 1-way ANOVA with Tukey's HSD.

\section{RESULTS}

\section{Physical variables}

We found seasonal differences in physical characteristics of the habitat types. Water depth was the most variable factor and was significantly lower in ME ( $\mathrm{p}<$ 0.001) than both OYS and SNB (Near and Far) over all sampling events (Table 3). Spring (May) water temperature and salinity differed among habitats with lower water temperatures in SNB (Near and Far) as compared to OYS $(p=0.042)$. OYS had significantly lower salinity than SNB ( $\mathrm{p}<0.001 ;$ Near and Far) and ME $(p=0.019)$. The only other differences among environ-

Table 3. Comparison of environmental characteristics among habitat types by seasons. Mean ( \pm SE) are given for variables measured in each habitat type that we sampled seasonally from May 2000 through February 2001. Refer to Table 1 for sample size of each mean and abbreviations. Resulting p-values are given for ANOVA analyses used to compare all habitat types (habitat effect) and 3 a priori contrasts testing different habitat combinations. ${ }^{*}$ ANOVA probability value was significant at the $5 \%$ level

\begin{tabular}{|c|c|c|c|c|c|}
\hline $\begin{array}{l}\text { Habitat } \\
\text { ANOVA p-values }\end{array}$ & Temperature $\left({ }^{\circ} \mathrm{C}\right)$ & Salinity & Depth $(\mathrm{cm})$ & $\begin{array}{c}\text { Dissolved } \\
\text { oxygen }\left(\mathrm{mg} \mathrm{l}^{-1}\right)\end{array}$ & Turbidity (FTU) \\
\hline \multicolumn{6}{|l|}{ May 2000} \\
\hline OYS & $27.1 \pm 0.38$ & $30.4 \pm 0.16$ & $56.7 \pm 1.01$ & $6.4 \pm 0.22$ & $19.4 \pm 7.01$ \\
\hline $\mathrm{ME}$ & $27.2 \pm 0.63$ & $31.4 \pm 0.27$ & $17.5 \pm 1.57$ & $5.7 \pm 0.31$ & $20.8 \pm 6.06$ \\
\hline SNB Near & $25.3 \pm 0.12$ & $34.1 \pm 0.11$ & $55.8 \pm 3.01$ & $5.8 \pm 0.08$ & $16.4 \pm 1.83$ \\
\hline SNB Far & $26.5 \pm 0.46$ & $31.4 \pm 0.40$ & $61.8 \pm 2.09$ & $6.3 \pm 0.17$ & $11.1 \pm 1.77$ \\
\hline Habitat effect & $0.027^{*}$ & $<0.001^{*}$ & $<0.001^{*}$ & 0.067 & 0.444 \\
\hline OYS vs. ME & 0.829 & 0.019 & $<0.001$ & & \\
\hline OYS vs. SNB & 0.042 & $<0.001$ & 0.402 & & \\
\hline SNB Near vs. SNB Far & 0.064 & $<0.001$ & 0.041 & & \\
\hline \multicolumn{6}{|l|}{ August 2000} \\
\hline OYS & $31.2 \pm 0.42$ & $37.4 \pm 0.48$ & $50.3 \pm 3.52$ & $5.8 \pm 0.32$ & $15.7 \pm 1.97$ \\
\hline ME & $31.2 \pm 0.60$ & $37.9 \pm 0.50$ & $16.5 \pm 2.96$ & $6.8 \pm 0.44$ & $19.8 \pm 3.41$ \\
\hline SNB Near & $31.7 \pm 0.51$ & $38.1 \pm 0.43$ & $40.9 \pm 2.95$ & $6.9 \pm 0.29$ & $16.0 \pm 1.69$ \\
\hline SNB Far & $31.3 \pm 0.47$ & $38.6 \pm 0.60$ & $65.2 \pm 3.12$ & $6.5 \pm 0.40$ & $16.2 \pm 1.75$ \\
\hline Habitat effect & 0.906 & 0.466 & $<0.001^{*}$ & 0.162 & 0.568 \\
\hline OYS vs. ME & & & $<0.001$ & & \\
\hline OYS vs. SNB & & & 0.477 & & \\
\hline SNB Near vs. SNB Far & & & $<0.001$ & & \\
\hline \multicolumn{6}{|l|}{ November 2000} \\
\hline OYS & $22.9 \pm 0.60$ & $30.3 \pm 0.34$ & $65.0 \pm 3.33$ & $7.1 \pm 0.12$ & $24.5 \pm 1.99$ \\
\hline ME & $22.4 \pm 0.69$ & $30.3 \pm 0.37$ & $24.7 \pm 2.85$ & $6.7 \pm 0.18$ & $16.7 \pm 2.46$ \\
\hline SNB Near & $22.4 \pm 0.47$ & $30.6 \pm 3.71$ & $59.2 \pm 1.96$ & $6.8 \pm 0.19$ & $15.9 \pm 2.77$ \\
\hline SNB Far & $21.5 \pm 0.45$ & $30.6 \pm 0.22$ & $67.9 \pm 2.05$ & $7.1 \pm 0.10$ & $19.1 \pm 1.26$ \\
\hline Habitat effect & 0.370 & 0.842 & $<0.001^{*}$ & 0.082 & $0.038^{*}$ \\
\hline OYS vs. ME & & & $<0.001$ & & 0.016 \\
\hline OYS vs. SNB & & & 0.644 & & 0.014 \\
\hline SNB Near vs. SNB Far & & & 0.024 & & 0.311 \\
\hline \multicolumn{6}{|l|}{ February 2001} \\
\hline OYS & $16.9 \pm 0.18$ & $20.1 \pm 0.10$ & $41.9 \pm 2.62$ & $8.8 \pm 0.23$ & $3.2 \pm 0.43$ \\
\hline ME & $13.7 \pm 0.15$ & $20.3 \pm 0.21$ & $15.6 \pm 1.12$ & $9.3 \pm 0.07$ & $6.9 \pm 0.96$ \\
\hline SNB Near & $12.9 \pm 0.05$ & $20.3 \pm 0.15$ & $31.8 \pm 2.51$ & $9.3 \pm 0.25$ & $5.6 \pm 0.96$ \\
\hline SNB Far & $13.0 \pm 0.05$ & $20.5 \pm 0.16$ & $46.1 \pm 4.22$ & $9.4 \pm 0.15$ & $4.5 \pm 0.77$ \\
\hline Habitat effect & $<0.001^{*}$ & 0.387 & $<0.001^{*}$ & 0.242 & $0.028^{*}$ \\
\hline OYS vs. ME & $<0.001$ & & $<0.001$ & & 0.006 \\
\hline OYS vs. SNB & $<0.001$ & & 0.432 & & 0.049 \\
\hline SNB Near vs. SNB Far & 0.729 & & 0.002 & & 0.295 \\
\hline
\end{tabular}


mental conditions were in winter samples (February), when water temperature and turbidity varied among habitats. Water temperature was significantly higher in OYS than in both ME $(\mathrm{p}<0.001)$ and SNB $(\mathrm{p}<0.001$; Near and Far). However, OYS had the lowest turbidity when compared to ME ( $p=0.006)$ and SNB $(p=0.049$; Near and Far) (Table 3).

\section{Nekton density and biomass}

We collected a total of 16177 organisms with a biomass of $7.76 \mathrm{~kg}$ representing 38 fish and 21 decapod crustacean species throughout the study period. The drop sampler performed efficiently in OYS, and our recovery rate was high. The mean recovery rate was $>96 \%$ for each species stocked, and no difference was detected in the recovery rates among species (ANOVA; $F=0.63 ; \mathrm{df}=3,36 ; \mathrm{p}=0.459$ ). This nekton recovery rate was similar to that for drop samples in marsh and on nonvegetated bottom (Zimmerman et al 1984, Rozas \& Minello 1997, Stunz et al. 2002a) supporting the validity of using drop samplers for these habitat comparisons.

Nekton abundance peaked in spring, when we collected a total of 5999 organisms (29 fish and 18 decapod crustacean species) and a total biomass of $3.55 \mathrm{~kg}$ wet wt. Fewer organisms (3233) and lower biomass $(1.80 \mathrm{~kg})$, representing 31 species of fish and 18 species of decapod crustaceans, were collected in summer (August). We collected 4391 organisms (21 fish and 24 decapod crustacean species) and a biomass of $1.91 \mathrm{~kg}$ in the fall (November). The fewest number of nekton (2771 organisms) and the lowest biomass $(0.50 \mathrm{~kg})$ were collected in winter. The fewest species were also collected in winter, representing only 17 fish and 11 decapod crustacean species. Decapod crustaceans outnumbered fishes in all seasons and represented $85,78,83$, and $52 \%$ of the total animals we collected in spring, summer, fall, and winter, respectively. Decapod crustaceans also dominated the seasonal biomass in spring $(72 \%)$, summer $(57 \%)$, fall (64\%), and winter (68\%). Pinfish, spot, naked goby Gobiosoma bosc, and bay anchovy Anchoa mitchilli were the most abundant fishes. The most abundant nektonic crustaceans were grass shrimp Palaeomonetes spp. and blue crab Callinectes sapidus, while the most abundant benthic crustaceans were green porcelain crab Petrolisthes armatus and snapping shrimp Alpheus heterochaelis (Table 4).

When we compared animal densities among all habitat types, relatively low densities occurred over SNB, thus data from SNB Near and SNB Far samples were combined for some analyses. Generally, highest densities of fishes occurred on intertidal OYS, and highest densities of decapod crustaceans occurred on OYS or in ME. Fish density was much greater on OYS during all seasons, especially in spring and summer, when densities were approximately $6 \times$ higher than in $\mathrm{ME}$ and SNB. Fish densities were more similar in fall, although still highest on OYS. The only exception was in winter when there was also a high number of fishes found over SNB (Fig. 2a). Decapod crustacean density patterns were similar, with the highest densities occurring on OYS in spring, summer, and winter, except in fall, when ME contained the highest densities of crustaceans (Fig. 2b).

In spring, the dominant fishes present (inland silverside, gulf toadfish, pinfish, and naked goby) were most abundant in structured habitats, with higher densities in OYS than in ME (see Table S1 in the supplement, available at www.int-res.com/articles/ suppl/m406p147_app.pdf). Bay anchovy had highest densities over SNB. All crustaceans had substantially higher densities in structured habitat (OYS and ME) compared with shallow open water, and nektonic crustaceans such as grass shrimp, brown shrimp, and blue crab had similar densities in OYS and ME, while green porcelain crab and snapping shrimp had highest densities in OYS. This pattern was similar in the summer, except that both white shrimp and brown shrimp had highest densities in ME compared with all other habitat types. In fall, Atlantic croaker were present in the samples with similar densities in OYS and SNB, while none occurred in ME. Mud crabs and snapping shrimp continued to have highest densities in OYS, but grass shrimp, blue crab, brown shrimp, white shrimp, and pink shrimp all had highest mean densities in ME habitat. During winter, fish densities were relatively low and similar among habitat types, except for high densities of pinfish in OYS (Table S1). Crustaceans also were less abundant during winter, but highest mean densities of most species present occurred in OYS.

Our analyses also showed that OYS and ME supported the highest biomass of fishes and crustaceans. The biomass of fishes was highest over OYS in spring and summer and in ME in fall and winter. The SNB also supported fairly high fish biomass in spring but rarely contributed in the other seasons (Fig. 2c). Crustacean biomass was greatest in OYS during spring, summer, and winter, followed by ME during the same seasons. In fall, crustacean biomass was highest in SNB, followed by OYS (Fig. 2d). In general, biomass patterns for these selected species closely tracked density patterns, and biomass values have been archived in Table S2 (in the supplement available at www.intres.com/articles/suppl/m406p147_app.pdf).

For selected species of interest, we examined the density, biomass, and length patterns over periods of 
Table 4. Overall mean densities (and $1 \mathrm{SE}$ ) as number $\mathrm{m}^{-2}$ of all collected fishes and crustaceans in 4 habitat types including OYS, ME, and SNB Near and SNB Far. Refer to Table 1 for sample size of each mean and abbreviations. The total number and relative abundance $($ rel. abun. $=$ no. ind./total no. ind. collected $\times 100)$ are also given

\begin{tabular}{|c|c|c|c|c|c|c|c|c|c|c|c|}
\hline \multirow{3}{*}{$\begin{array}{l}\text { Common } \\
\text { name }\end{array}$} & \multirow{3}{*}{$\begin{array}{c}\text { Scientific } \\
\text { name }\end{array}$} & \multirow{3}{*}{$\begin{array}{c}\text { Total } \\
\text { no. }\end{array}$} & \multirow{3}{*}{$\begin{array}{l}\text { Rel. } \\
\text { abun. } \\
(\%)\end{array}$} & \multicolumn{2}{|c|}{ OYS } & \multicolumn{2}{|c|}{ ME } & \multicolumn{4}{|c|}{$-\mathrm{SNB}$} \\
\hline & & & & \multirow[t]{2}{*}{ Mean } & \multirow[t]{2}{*}{ SE } & \multirow[t]{2}{*}{ Mean } & \multirow[t]{2}{*}{ SE } & \multicolumn{2}{|c|}{ Near } & \multicolumn{2}{|c|}{ Far } \\
\hline & & & & & & & & Mean & $\mathrm{SE}$ & Mean & SE \\
\hline Total fishes & & 3791 & 22.8 & 17.22 & $(1.88)$ & 4.02 & $(0.69)$ & 7.60 & $(1.60)$ & 6.54 & $(1.80)$ \\
\hline Pinfish & Lagodon rhomboides & 839 & 5.2 & 4.08 & (1.13) & 0.52 & $(0.18)$ & 1.22 & $(0.64)$ & 2.21 & $(1.41)$ \\
\hline Spot & Leiostomus xanthurus & 371 & 2.3 & 0.24 & $(0.11)$ & 0.20 & $(0.12)$ & 2.61 & $(1.24)$ & 0.57 & $(0.22)$ \\
\hline Naked goby & Gobiosoma bosc & 366 & 2.3 & 3.43 & $(0.71)$ & 0.03 & $(0.02)$ & 0.02 & $(0.01)$ & 0.04 & $(0.04)$ \\
\hline Bay anchovy & Anchoa mitchilli & 321 & 2.0 & 0.24 & $(0.15)$ & 0.00 & $(0.00)$ & 1.44 & $(0.66)$ & 1.37 & $(0.47)$ \\
\hline Darter goby & Gobionellus boleosoma & 302 & 1.9 & 1.00 & $(0.25)$ & 1.43 & $(0.44)$ & 0.43 & $(0.14)$ & 0.15 & $(0.06)$ \\
\hline Gulf toadfish & Opsanus beta & 286 & 1.8 & 2.73 & $(1.02)$ & 0.02 & $(0.01)$ & 0.00 & $(0.00)$ & 0.00 & $(0.00)$ \\
\hline Inland silverside & Menidia beryllina & 269 & 1.7 & 1.75 & $(1.08)$ & 0.28 & $(0.20)$ & 0.51 & $(0.26)$ & 0.07 & $(0.05)$ \\
\hline Goby species & Gobiidae unid. & 133 & 0.8 & 1.06 & $(0.65)$ & 0.01 & $(0.01)$ & 0.16 & $(0.12)$ & 0.05 & $(0.03)$ \\
\hline Atlantic croaker & Micropogonias undulatus & 124 & 0.8 & 0.40 & $(0.22)$ & 0.01 & $(0.01)$ & 0.48 & $(0.19)$ & 0.29 & $(0.10)$ \\
\hline Sheepshead minnow & w Cyprinodon variegatus & 79 & 0.5 & 0.02 & $(0.01)$ & 0.30 & $(0.09)$ & 0.14 & $(0.07)$ & 0.31 & $(0.27)$ \\
\hline Clown goby & Microgobius gulosus & 71 & 0.4 & 0.22 & $(0.09)$ & 0.02 & $(0.02)$ & 0.10 & $(0.05)$ & 0.33 & $(0.10)$ \\
\hline Longnose killifish & Fundulus similis & 68 & 0.4 & 0.00 & $(0.00)$ & 0.15 & $(0.07)$ & 0.00 & $(0.00)$ & 0.49 & (0.49) \\
\hline Blackcheek tonguefi & fish Symphurus plagiusa & 54 & 0.3 & 0.33 & $(0.09)$ & 0.03 & $(0.02)$ & 0.14 & $(0.05)$ & 0.03 & $(0.02)$ \\
\hline Gulf menhaden & Brevoortia patronus & 43 & 0.3 & 0.01 & $(0.01)$ & 0.33 & $(0.32)$ & 0.04 & $(0.04)$ & 0.05 & $(0.03)$ \\
\hline Code goby & Gobiosoma robustum & 41 & 0.3 & 0.14 & $(0.07)$ & 0.06 & $(0.04)$ & 0.02 & $(0.02)$ & 0.16 & $(0.07)$ \\
\hline Seatrout species & Cynoscion sp. & 34 & 0.2 & 0.27 & $(0.27)$ & 0.01 & $(0.01)$ & 0.00 & $(0.00)$ & 0.05 & $(0.05)$ \\
\hline Skilletfish & Gobiesox strumosus & 27 & 0.2 & 0.26 & $(0.08)$ & 0.00 & $(0.00)$ & 0.00 & $(0.00)$ & 0.00 & $(0.00)$ \\
\hline Sciaenidae species & Sciaenidae unid. & 22 & 0.1 & 0.17 & $(0.10)$ & 0.01 & $(0.01)$ & 0.00 & $(0.00)$ & 0.03 & $(0.02)$ \\
\hline Bay whiff & Citharichthys spilopterus & 18 & 0.1 & 0.06 & $(0.03)$ & 0.02 & $(0.02)$ & 0.04 & $(0.02)$ & 0.05 & $(0.02)$ \\
\hline Pigfish & Orthopristis chrysoptera & 15 & 0.1 & 0.12 & $(0.05)$ & 0.00 & $(0.00)$ & 0.01 & $(0.01)$ & 0.02 & $(0.01)$ \\
\hline Silver perch & Bairdiella chrysoura & 14 & 0.1 & 0.10 & $(0.06)$ & 0.00 & $(0.00)$ & 0.04 & $(0.03)$ & 0.00 & $(0.00)$ \\
\hline Speckled eel worm & Myrophis punctatus & 14 & 0.1 & 0.11 & $(0.04)$ & 0.02 & $(0.02)$ & 0.00 & $(0.00)$ & 0.01 & $(0.01)$ \\
\hline Sheepshead $\quad A$ & Archosargus probatocephalus & 13 & 0.1 & 0.11 & $(0.03)$ & 0.02 & $(0.01)$ & 0.00 & $(0.00)$ & 0.00 & $(0.00)$ \\
\hline Striped mullet & Mugil cephalus & 13 & 0.1 & 0.00 & $(0.00)$ & 0.00 & $(0.00)$ & 0.04 & $(0.02)$ & 0.08 & $(0.06)$ \\
\hline Red drum & Sciaenops ocellatus & 13 & 0.1 & 0.00 & $(0.00)$ & 0.11 & $(0.06)$ & 0.01 & $(0.01)$ & 0.01 & $(0.01)$ \\
\hline Lined sole & Achirus lineatus & 12 & 0.1 & 0.01 & $(0.01)$ & 0.07 & $(0.03)$ & 0.04 & $(0.02)$ & 0.00 & $(0.00)$ \\
\hline Spotted seatrout & Cynoscion nebulosus & 12 & 0.1 & 0.01 & $(0.01)$ & 0.06 & $(0.03)$ & 0.04 & $(0.02)$ & 0.01 & $(0.01)$ \\
\hline Gulf killifish & Fundulus grandis & 12 & 0.1 & 0.00 & $(0.00)$ & 0.12 & $(0.04)$ & 0.00 & $(0.00)$ & 0.00 & $(0.00)$ \\
\hline Southern flounder & Paralichthys lethostigma & 11 & 0.1 & 0.04 & $(0.02)$ & 0.03 & $(0.02)$ & 0.00 & $(0.00)$ & 0.04 & $(0.02)$ \\
\hline Unidentified fish & Unid. fish & 10 & 0.1 & 0.08 & $(0.03)$ & 0.02 & $(0.02)$ & 0.00 & $(0.00)$ & 0.00 & $(0.00)$ \\
\hline Mojarra species & Gerreidae unid. & 8 & 0.0 & 0.07 & $(0.07)$ & 0.01 & $(0.01)$ & 0.00 & $(0.00)$ & 0.00 & $(0.00)$ \\
\hline Flagfin mojarra & Eucinostomus melanopterus & 8 & 0.0 & 0.02 & $(0.02)$ & 0.00 & $(0.00)$ & 0.03 & $(0.02)$ & 0.02 & $(0.01)$ \\
\hline Mojarra species & Eucinostomus sp. & 8 & 0.0 & 0.02 & $(0.02)$ & 0.00 & $(0.00)$ & 0.02 & $(0.01)$ & 0.03 & $(0.02)$ \\
\hline Green goby & Microgobius thalassinus & 8 & 0.0 & 0.05 & $(0.03)$ & 0.00 & $(0.00)$ & 0.00 & $(0.00)$ & 0.00 & $(0.00)$ \\
\hline Diamond killifish & Adinia xenica & 8 & 0.0 & 0.00 & $(0.00)$ & 0.03 & $(0.02)$ & 0.00 & $(0.00)$ & 0.00 & $(0.00)$ \\
\hline Striped blenny & Chasmodes bosquianus & 8 & 0.0 & 0.03 & $(0.02)$ & 0.00 & $(0.00)$ & 0.00 & $(0.00)$ & 0.00 & $(0.00)$ \\
\hline Anchovy species & Engraulidae unid. & 8 & 0.0 & 0.01 & $(0.01)$ & 0.00 & $(0.00)$ & 0.01 & $(0.01)$ & 0.01 & $(0.01)$ \\
\hline Spanish sardine & Sardinella aurita & 8 & 0.0 & 0.00 & $(0.00)$ & 0.00 & $(0.00)$ & 0.00 & $(0.00)$ & 0.03 & $(0.03)$ \\
\hline Clupeidae species & Clupeidae unid. & 8 & 0.0 & 0.01 & $(0.01)$ & 0.01 & $(0.01)$ & 0.00 & $(0.00)$ & 0.00 & $(0.00)$ \\
\hline Goby species & Gobiosoma sp. & 8 & 0.0 & 0.02 & $(0.02)$ & 0.00 & $(0.00)$ & 0.00 & $(0.00)$ & 0.00 & $(0.00)$ \\
\hline Grey snapper & Lutjanus griseus & 8 & 0.0 & 0.01 & $(0.01)$ & 0.01 & $(0.01)$ & 0.00 & $(0.00)$ & 0.00 & $(0.00)$ \\
\hline White mullet & Mugil curema & 8 & 0.0 & 0.00 & $(0.00)$ & 0.02 & $(0.01)$ & 0.00 & $(0.00)$ & 0.00 & $(0.00)$ \\
\hline Killifish species & Fundulidae unid. & 8 & 0.0 & 0.00 & $(0.00)$ & 0.01 & $(0.01)$ & 0.00 & $(0.00)$ & 0.00 & $(0.00)$ \\
\hline Silverside species & Menidia sp. & 8 & 0.0 & 0.01 & $(0.01)$ & 0.00 & $(0.00)$ & 0.00 & $(0.00)$ & 0.00 & $(0.00)$ \\
\hline Goby species & Microgobius sp. & 8 & 0.0 & 0.01 & $(0.01)$ & 0.00 & $(0.00)$ & 0.00 & $(0.00)$ & 0.00 & $(0.00)$ \\
\hline Mullet species & Mugilidae unid. & 8 & 0.0 & 0.00 & $(0.00)$ & 0.00 & $(0.00)$ & 0.00 & $(0.00)$ & 0.01 & $(0.01)$ \\
\hline Worm eels & Ophichthidae unid. & 8 & 0.0 & 0.00 & $(0.00)$ & 0.00 & $(0.00)$ & 0.00 & $(0.00)$ & 0.01 & $(0.01)$ \\
\hline Crested cusk eel & Ophidion welshi & 8 & 0.0 & 0.00 & $(0.00)$ & 0.00 & $(0.00)$ & 0.00 & $(0.00)$ & 0.01 & $(0.01)$ \\
\hline Least puffer & Sphoeroides parvus & 8 & 0.0 & 0.00 & $(0.00)$ & 0.00 & $(0.00)$ & 0.01 & $(0.01)$ & 0.00 & $(0.00)$ \\
\hline Poecilidae species & Poecilidae unid. & 8 & 0.0 & 0.00 & $(0.00)$ & 0.01 & $(0.01)$ & 0.00 & $(0.00)$ & 0.00 & $(0.00)$ \\
\hline Black drum & Pogonias cromis & 8 & 0.0 & 0.00 & $(0.00)$ & 0.00 & $(0.00)$ & 0.01 & $(0.01)$ & 0.00 & $(0.00)$ \\
\hline Gulf pipefish & Syngnathus scovelli & 8 & 0.0 & 0.00 & $(0.00)$ & 0.01 & $(0.01)$ & 0.00 & $(0.00)$ & 0.00 & $(0.00)$ \\
\hline Inshore lizardfish & Synodus foetens & 8 & 0.0 & 0.00 & $(0.00)$ & 0.00 & $(0.00)$ & 0.00 & $(0.00)$ & 0.01 & $(0.01)$ \\
\hline
\end{tabular}


Table 4 (continued)

\begin{tabular}{|c|c|c|c|c|c|c|c|c|c|c|c|}
\hline \multirow{3}{*}{$\begin{array}{l}\text { Common } \\
\text { name }\end{array}$} & \multirow{3}{*}{$\begin{array}{c}\text { Scientific } \\
\text { name }\end{array}$} & \multirow{3}{*}{$\begin{array}{c}\text { Total } \\
\text { no. }\end{array}$} & \multirow{3}{*}{$\begin{array}{l}\text { Rel. } \\
\text { abun. } \\
(\%)\end{array}$} & \multicolumn{2}{|c|}{ OYS } & \multicolumn{2}{|c|}{$\mathrm{ME}$} & \multicolumn{4}{|c|}{$-\mathrm{SNB}$} \\
\hline & & & & \multirow[t]{2}{*}{ Mean } & \multirow[t]{2}{*}{ SE } & \multirow[t]{2}{*}{ Mean } & \multirow[t]{2}{*}{ SE } & \multicolumn{2}{|c|}{ Near } & \multicolumn{2}{|c|}{ Far } \\
\hline & & & & & & & & Mean & SE & Mean & SE \\
\hline Total crustaceans & & 12386 & 77.2 & 62.27 & $(9.90)$ & 55.03 & $(8.80)$ & 5.05 & $(0.84)$ & 4.08 & $(1.42)$ \\
\hline Grass shrimp species & Palaemonetes sp. & 3597 & 22.4 & 13.76 & $(2.14)$ & 20.85 & $(4.94)$ & 0.95 & $(0.47)$ & 0.59 & $(0.30)$ \\
\hline Daggerblade grass shrimp & ip Palaemonetes pugio & 1531 & 9.5 & 3.95 & $(0.87)$ & 10.40 & $(2.78)$ & 0.35 & $(0.18)$ & 0.78 & $(0.54)$ \\
\hline Green porcelain crab & Petrolisthes armatus & 1418 & 8.8 & 13.09 & $(4.29)$ & 0.07 & $(0.04)$ & 0.12 & $(0.05)$ & 0.35 & $(0.22)$ \\
\hline Blue crab & Callinectes sapidus & 1194 & 7.4 & 3.13 & $(0.49)$ & 6.26 & (1.91) & 1.52 & $(0.35)$ & 1.03 & $(0.26)$ \\
\hline Snapping shrimp & Alpheus heterochaelis & 1010 & 6.3 & 9.46 & $(1.56)$ & 0.08 & $(0.04)$ & 0.08 & $(0.05)$ & 0.09 & $(0.08)$ \\
\hline Brown shrimp & Farfantepenaeus aztecus & 960 & 6.0 & 2.30 & $(0.68)$ & 6.15 & (1.29) & 0.83 & $(0.17)$ & 0.41 & $(0.10)$ \\
\hline Depressed mud crab & Eurypanopeus depressus & 530 & 3.3 & 5.10 & $(1.02)$ & 0.00 & $(0.00)$ & 0.00 & $(0.00)$ & 0.00 & $(0.00)$ \\
\hline Mud crabs & Xanthidae unid. & 528 & 3.3 & 4.95 & $(0.90)$ & 0.00 & $(0.00)$ & 0.00 & $(0.00)$ & 0.12 & $(0.11)$ \\
\hline Atlantic mud crab & Panopeus herbstii & 321 & 2.0 & 2.85 & $(0.68)$ & 0.02 & $(0.01)$ & 0.00 & $(0.00)$ & 0.21 & $(0.18)$ \\
\hline Marsh grass shrimp & Palaemonetes vulgaris & 284 & 1.8 & 1.32 & $(0.53)$ & 1.46 & $(0.79)$ & 0.06 & $(0.04)$ & 0.01 & $(0.01)$ \\
\hline White shrimp & Litopenaeus setiferus & 253 & 1.6 & 0.20 & $(0.07)$ & 2.04 & $(0.78)$ & 0.33 & $(0.22)$ & 0.03 & $(0.02)$ \\
\hline Pink shirmp & Farfantepenaeus duorarum & n 181 & 1.1 & 0.38 & $(0.10)$ & 0.87 & $(0.19)$ & 0.39 & $(0.10)$ & 0.16 & $(0.05)$ \\
\hline Striped hermit crab & Clibanarius vittatus & 145 & 0.9 & 0.15 & $(0.06)$ & 1.17 & $(0.40)$ & 0.13 & $(0.04)$ & 0.03 & $(0.02)$ \\
\hline Gulf Marsh fiddler crab & Uca longisignalis & 81 & 0.5 & 0.00 & $(0.00)$ & 0.84 & $(0.32)$ & 0.00 & $(0.00)$ & 0.00 & $(0.00)$ \\
\hline Brackish grass shrimp $P$ & Palaemonetes intermedius & 74 & 0.5 & 0.13 & $(0.09)$ & 0.62 & $(0.38)$ & 0.00 & $(0.00)$ & 0.00 & $(0.00)$ \\
\hline Portunidae species & Portunidae unid. & 60 & 0.4 & 0.11 & $(0.07)$ & 0.51 & $(0.37)$ & 0.00 & $(0.00)$ & 0.00 & $(0.00)$ \\
\hline Callinectes species & Callinectes sp. & 54 & 0.3 & 0.48 & $(0.19)$ & 0.00 & $(0.00)$ & 0.03 & $(0.02)$ & 0.01 & $(0.01)$ \\
\hline Stone crab & Menippe mercenaria & 48 & 0.3 & 0.46 & $(0.10)$ & 0.00 & $(0.00)$ & 0.00 & $(0.00)$ & 0.00 & $(0.00)$ \\
\hline Lesser blue crab & Callinectes similis & 29 & 0.2 & 0.14 & $(0.09)$ & 0.00 & $(0.00)$ & 0.07 & $(0.03)$ & 0.06 & $(0.03)$ \\
\hline Ridgeback mud crab & Panopeus turgidus & 24 & 0.1 & 0.03 & $(0.02)$ & 0.00 & $(0.00)$ & 0.21 & $(0.21)$ & 0.00 & $(0.00)$ \\
\hline Fiddler crab species & $U_{C a} \mathrm{sp}$ & 15 & 0.1 & 0.04 & $(0.03)$ & 0.11 & $(0.06)$ & 0.00 & $(0.00)$ & 0.00 & $(0.00)$ \\
\hline Xanthid mud crab & Rhithropanopeus harrisii & 12 & 0.1 & 0.03 & $(0.02)$ & 0.02 & $(0.01)$ & 0.00 & $(0.00)$ & 0.06 & $(0.06)$ \\
\hline- & Ambidexter symmetricus & 5 & 0.0 & 0.05 & $(0.03)$ & 0.00 & $(0.00)$ & 0.00 & $(0.00)$ & 0.00 & $(0.00)$ \\
\hline Porcelain crab & Petrolisthes sp. & 5 & 0.0 & 0.05 & $(0.05)$ & 0.00 & $(0.00)$ & 0.00 & $(0.00)$ & 0.00 & $(0.00)$ \\
\hline Marsh crab & Sesarma reticulatum & 5 & 0.0 & 0.00 & $(0.00)$ & 0.05 & $(0.03)$ & 0.00 & $(0.00)$ & 0.00 & $(0.00)$ \\
\hline Sargassum shrimp & Latreutes parvulus & 4 & 0.0 & 0.01 & $(0.01)$ & 0.01 & $(0.01)$ & 0.00 & $(0.00)$ & 0.02 & $(0.02)$ \\
\hline Long-clawed hermit crab & Pagurus longicarpus & 4 & 0.0 & 0.02 & $(0.02)$ & 0.01 & $(0.01)$ & 0.00 & $(0.00)$ & 0.01 & $(0.01)$ \\
\hline Diogenidae species & Diogenidae unid. & 2 & 0.0 & 0.02 & $(0.01)$ & 0.00 & $(0.00)$ & 0.00 & $(0.00)$ & 0.00 & $(0.00)$ \\
\hline Pinnixia species & Pinnixa sp. & 2 & 0.0 & 0.00 & $(0.00)$ & 0.00 & $(0.00)$ & 0.00 & $(0.00)$ & 0.02 & $(0.01)$ \\
\hline Aviu shrimp & Acetes americanus & 1 & 0.0 & 0.01 & $(0.01)$ & 0.00 & $(0.00)$ & 0.00 & $(0.00)$ & 0.00 & $(0.00)$ \\
\hline Decapod species & Decapoda unid. & 1 & 0.0 & 0.01 & $(0.01)$ & 0.00 & $(0.00)$ & 0.00 & $(0.00)$ & 0.00 & $(0.00)$ \\
\hline Combclaw shrimp & Leptochela serratorbita & 1 & 0.0 & 0.01 & $(0.01)$ & 0.00 & $(0.00)$ & 0.00 & $(0.00)$ & 0.00 & $(0.00)$ \\
\hline Spider crab & Libinia dubia & 1 & 0.0 & 0.01 & $(0.01)$ & 0.00 & $(0.00)$ & 0.00 & $(0.00)$ & 0.00 & $(0.00)$ \\
\hline Estuarine longeye shrimp & Ogyrides alphaerostris & 1 & 0.0 & 0.00 & $(0.00)$ & 0.00 & $(0.00)$ & 0.00 & $(0.00)$ & 0.01 & $(0.01)$ \\
\hline Banded porcelain crab & Petrolisthes galathinus & 1 & 0.0 & 0.01 & $(0.01)$ & 0.00 & $(0.00)$ & 0.00 & $(0.00)$ & 0.00 & $(0.00)$ \\
\hline Porcelain crab species & Porcellanidae unid. & 1 & 0.0 & 0.01 & $(0.01)$ & 0.00 & $(0.00)$ & 0.00 & $(0.00)$ & 0.00 & $(0.00)$ \\
\hline Arrow shrimp & Tozeuma carolinense & 1 & 0.0 & 0.01 & $(0.01)$ & 0.00 & $(0.00)$ & 0.00 & $(0.00)$ & 0.00 & $(0.00)$ \\
\hline Mud crab species & Upogebia sp. & 1 & 0.0 & 0.01 & $(0.01)$ & 0.00 & $(0.00)$ & 0.00 & $(0.00)$ & 0.00 & $(0.00)$ \\
\hline Grass shrimp species & Palaemonidae unid. & 1 & 0.0 & 0.00 & $(0.00)$ & 0.01 & $(0.01)$ & 0.00 & $(0.00)$ & 0.00 & $(0.00)$ \\
\hline
\end{tabular}

peak recruitment (Fig. 3). Red drum occurred only in $\mathrm{ME}$ and $\mathrm{SNB}$, and we did not find significant density differences $(p=0.486)$. Pinfish were most abundant on OYS, brown shrimp and white shrimp were highest in $\mathrm{ME}$, and blue crab were most abundant in ME and OYS (Fig. 3a). Biomass patterns for these species were similar, except red drum and white shrimp biomass were not different among habitats $(p=0.054$ and 0.119, respectively; Fig. 3b). Pinfish and blue crab exhibited significantly different sizes among habitats. Blue crabs were largest on OYS and ME and smallest over SNB. Brown shrimp and white shrimp were similar in size $(p=0.017$ and 0.191, respectively; no difference was found with Tukey's post hoc test for brown shrimp) among all habitats and red drum did not occur on OYS and their size did not differ between ME and SNB ( $p=0.691$; Fig. 3c).

\section{Species richness}

OYS habitat supported very high species richness in all seasons. The mean number of species in samples was significantly greater in OYS during spring $(\mathrm{p}<$ $0.001)$, summer $(\mathrm{p}<0.001)$, and fall $(\mathrm{p}<0.001)$ than in either ME or SNB Near and SNB Far (Fig. 4). Overall, species richness was much lower during winter, and OYS had similar richness to SNB Far; both of these habitat types had significantly higher species richness than ME and SNB Near (Fig. 4). 

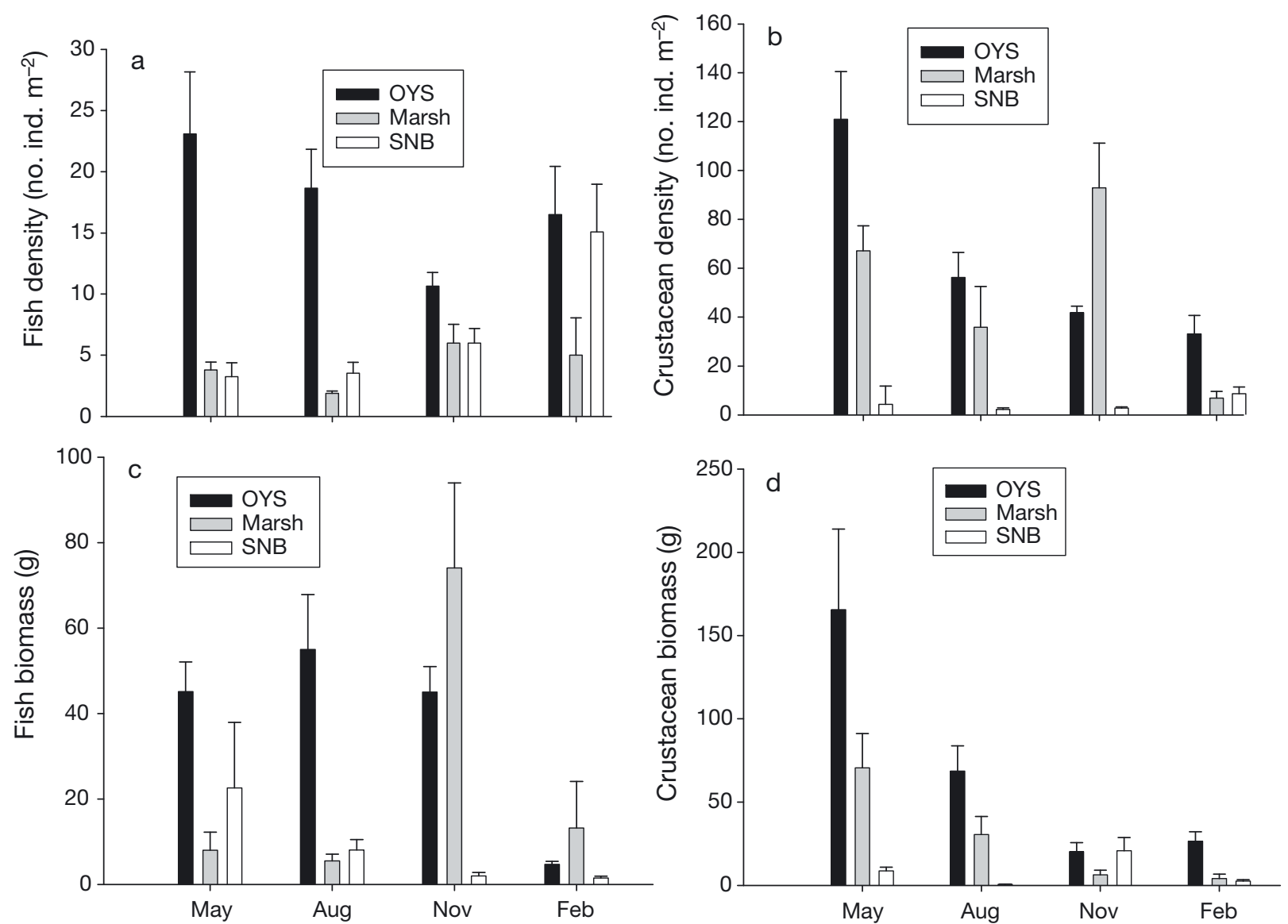

Fig. 2. Overall mean seasonal densities of (a) fish and (b) decapod crustaceans for each habitat, and mean seasonal biomass of (c) fish and (d) decapod crustaceans for each habitat. Refer to Table 1 for sample size of each density and biomass mean. Error bars indicate SE. OYS: oyster reef, SNB: shallow nonvegetated bottom

\section{DISCUSSION}

Our objective in the present study was to assess the relative value of OYS for estuarine nekton as compared to ME and SNB. Gear selectivity, bias, and efficiency have been central issues in attempts to sample nekton over OYS habitat (see Zimmerman et al. 1989, Wenner et al.1996, Minello 1999, Peterson et al. 2003, Coen et al. 2007), primarily due to the complex nature of the reef structure. We used a drop sampler to estimate nekton densities on isolated OYS plots, and our recovery trials showed that nekton could be efficiently collected from drop samples enclosing these highrelief, live OYS plots. Across all seasons, OYS supported a higher abundance and biomass of most nekton and greater species richness than ME and SNB. Thus, these data underscore the value of OYS and nekton habitat in estuarine ecosystems.

We found statistically significant differences in temperature, depth, and salinity among habitat types, but these differences were typically small, seasonally consistent, and unlikely to be biologically significant. The habitat types were located in close proximity facilitating potential movement among areas. However, several investigators have shown behavioral nekton responses to a variety of environmental variables (Wannamaker \& Rice 2000, McNatt \& Rice 2004), and the response of nekton to changes in these environmental variables warrants further examination.

Overall, OYS had a higher abundance of fishes than $\mathrm{ME}$ or $\mathrm{SNB}$, and this pattern was relatively consistent among species. Some exceptions to the pattern included spot and bay anchovy, which had highest densities over SNB. Red drum Sciaenops ocellatus occurred at relatively low abundance and were found in only ME and SNB. The highest densities of crustaceans were on structured habitats (OYS and ME), but differences between these 2 habitats were speciesspecific. Benthic species such as green porcelain crab, depressed mud crab, Atlantic mud crab, mud crab (Xanthidae), and snapping shrimp generally had significantly higher densities in OYS than in ME habitat. In contrast, nektonic crustaceans such as brown shrimp, white shrimp, grass shrimp, and blue crabs 


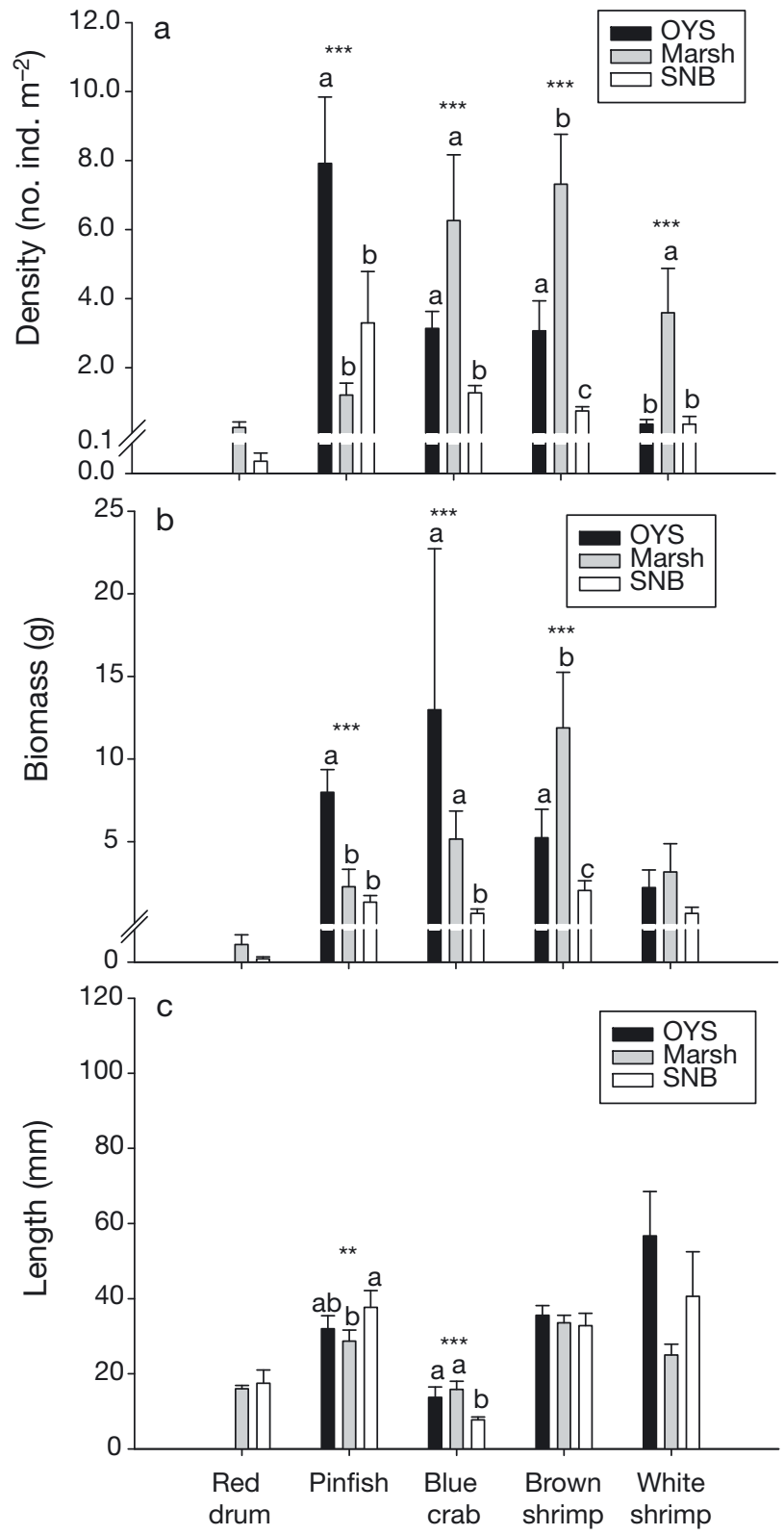

Fig. 3. Mean densities of selected fishes and crustaceans collected in OYS, ME, and SNB during their peak recruitment season (see Fig. 1, Table 1 for site descriptions). SNB Near and SNB Far were combined into SNB for analysis. Separate 1-way ANOVAs with season block were used to determine (a) density, (b) biomass, and (c) length differences among habitats for each species. Habitats for each species that share a common letter above the bar are not statistically different. Error bars indicate SE. Mean density and biomass were calculated for red drum from November 2000; pinfish from May 2000 and February 2001; blue crab from all sampling dates; brown shrimp from May, August, and November 2000; and white shrimp from August and November 2000. Refer to Table 1 for sample size of each density and biomass mean. Mean length sample size (n) for each species is as follows: red drum, oyster $=0$, marsh $=3$, $\mathrm{SNB}=2$; pinfish, $\mathrm{OYS}=20$, marsh $=12, \mathrm{SNB}=20$; blue crab, OYS $=35$, marsh $=29$, SNB $=49$; brown shrimp, OYS $=22$, marsh $=29, \mathrm{SNB}=33$; white shrimp, $\mathrm{OYS}=8, \operatorname{marsh}=13$,

$$
\mathrm{SNB}=8{ }^{* *} \mathrm{p}<0.01,{ }^{* * *} \mathrm{p}<0.001
$$

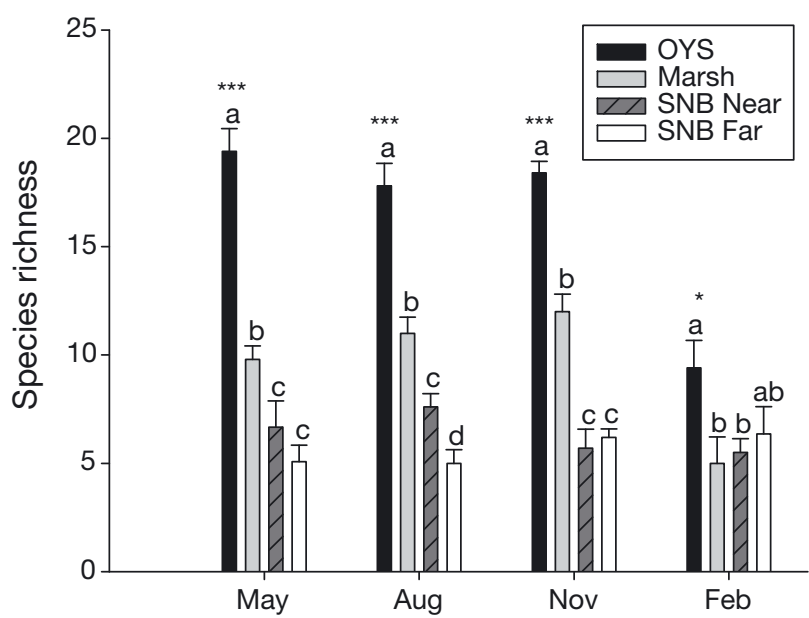

Fig. 4. Mean species richness among habitats seasonally. Refer to Table 1 for site descriptions and habitat sample sizes (n) for each date. Separate 1-way ANOVAs were used to determine species richness differences among habitats for each date. Habitats for each date that share a common letter above the bar are similar. Error bars indicate SE. ${ }^{*} \mathrm{p}<0.05$, ${ }^{* * *} \mathrm{p}<0.001$

had highest mean densities in ME, although differences between ME and OYS were not always significant. Patterns observed for biomass were very similar to those for density.

Many decapod crustaceans and fishes are known to use the marsh surface when the emergent vegetation is flooded and accessible to nekton (Kneib 1984, Baltz et al. 1993, Minello 1999). The high nekton densities we observed for many species in structured habitats such as OYS and ME indicate that value may be comparable for these habitats, but SNB may be more important for certain species. Our comparison of SNB Near and SNB Far was designed to examine this question. Bay anchovy Anchoa mitchilli was one of the most abundant species over nonvegetated bottom, but this species showed an inconsistent pattern of abundance between the 2 SNB habitat types. In spring, densities were highest in SNB Near, and in summer they were highest in SNB Far. Brown shrimp in spring and blue crab in summer also were more abundant in SNB Near than SNB Far; but the spatial resolution of our habitat categories may have reduced our ability to detect such associations, because Minello et al. (2003) showed that brown shrimp, white shrimp, and blue crab densities on SNB were highest within a few meters of the ME. Moreover, edge effects or habitat heterogeneity of structured habitat types at larger spatial scales may have influenced species abundance. Other studies have shown that spatial synergy and proximity of habitat types within the estuary can influence distribution patterns (Robblee \& Zieman 1984, Rozas \& Odum 1987, Rozas \& Minello 2006, Gain 2009). Irlandi \& Crawford (1997) showed that pinfish were over $2 \times$ as abundant 
within seagrass beds near marsh than in seagrass beds adjacent to SNB, and Rozas \& Minello (2006) found Harris mud crab Rhithropanopeus harrisii, rainwater killifish Lucania parva, and speckled worm eel Myrophis punctatus more abundant in submerged aquatic vegetation (SAV) near ME. Gain (2009) found higher abundance of decapod crustacean on OYS that was adjacent to SAV. These data suggest certain ecological advantages of being associated with habitats that are nearby vegetated shorelines.

A relationship of mean size and habitat use may reflect differences in recruitment, growth, or mortality or ontogenetic shifts in habitat selection (Sogard 1997, Stunz et al. 2002b, Rozas \& Minello 2006). Differences in mean size among habitats were not readily apparent for most species examined. However, blue crab size was significantly different among habitat types, with the largest crabs found in OYS and ME. These habitats also supported the highest densities of blue crab. These data suggest either a growth difference among habitat types or potential ontogenetic habitat selection shifts. Shervette \& Gelwick 2008 found elevated growth rates for white shrimp in oyster habitat over ME and SNB, and enhanced growth in ME habitat over SNB has been demonstrated for a few nekton species (Stunz et al. 2002b, Minello et al. 2003). Because of the proximity of the habitats at our study site and the mobility of blue crabs, the size difference we observed among habitats may simply be due to size-related habitat selection.

Species richness in OYS habitat was significantly higher than in other habitats during all seasons. Species richness was relatively low during winter when many species migrate out of the estuary. Harding \& Mann (1999) also showed more resident and transient species in OYS than over SNB, and Lenihan et al. (2001) reported species richness to be 2 to $3 \times$ higher on OYS than adjacent SNB. Moreover, many of the organisms using OYS do not occur on adjacent habitat types (Coen et al. 1999). In Galveston Bay, OYS provides some of the only subtidal structured habitat, and thus this availability may explain the richness patterns (Eggleston et al. 1998). Detailed analyses of community structure were well beyond the scope of this manuscript. However, further studies are warranted assessing the different assemblages using these habitat types, and our data clearly point to differences in the nekton and decapod crustacean community structure on OYS.

Given the complex nature of the reef structure, gear selectivity, bias, and efficiency are unavoidable, and may have affected this study and previous attempts to sample nekton over OYS habitat (Zimmerman et al. 1989, Wenner et al. 1996, Minello 1999, Peterson et al. 2003, Coen et al. 2007). To address any efficiency biases, we examined recovery rates from our oyster drop sampler to assess gear constraints. We were able to capture an average of $96 \%$ (and routinely $100 \%$ ) of our marked animals over OYS, demonstrating the efficiency and effectiveness of our gear. Previous studies in $\mathrm{ME}, \mathrm{SAV}$, and SNB have shown similar recovery rates (see Rozas \& Minello 1997 for review); thus, this gear appears to be an appropriate sampling device for shallow estuarine habitat types including OYS, when a seal can be made with the sediment and the sampler completely drained. However, the drop sampler is generally limited to depth $<1 \mathrm{~m}$, and the coverage area sampled is relatively small ( 1 to $2.6 \mathrm{~m}^{2}$ ). For open-water deep reefs, very mobile nekton, and those species that occur at very low densities, alternative gear types should be considered. In addition, to allow for the drop sampler to seal over mud bottom, the reef plots were slightly smaller than the diameter of the enclosure. Since OYS did not completely cover the plot, we may have potentially underestimated abundances. This design also creates a smaller, somewhat isolated plot with a high edge-perimeter ratio compared to a 'natural' oyster bed with a large interior. The high edge-perimeter ratio may artificially elevate abundance for small animals, but, conversely, the reduced plot size may restrict use by larger more mobile nekton. However, despite these limitations, we suggest the advantage of this method outweighs the potential biases, and the gear is effective for sampling OYS and other estuarine habitat types under many circumstances.

Several studies have described the habitat value of OYS and documented high abundance of estuarine nekton associated with this habitat (see review by Coen et al. 1999, and Coen et al. 2007). However, ours is one of the few studies to make direct comparisons among OYS and other habitat types. Although our use of manipulated oyster plots may have affected our results, these plots were within or adjacent to natural intertidal oyster beds, and they were composed of relatively high-relief live oysters. We believe that our estimates of nekton use are conservative, and that undisturbed portions of the reef would harbor at least these numbers of fishes and crustaceans. Peterson et al. (2003) concluded that OYS enhanced fish production over nearby nonvegetated areas, and several studies have shown elevated nekton abundance over oyster shell accumulations (Szedlmayer \& Howe 1997, Steimle \& Zetlin 2000, Lehnert \& Allen 2002, Shervette $\&$ Gelwick 2008). Several laboratory studies have documented that fish preferentially select for OYS (Posey et al. 1999, Stunz et al. 2001), and nekton may incur lower mortality in this structured habitat (Stunz et al. 2002a, Grabowski 2004) compared with other estuarine habitat types. Additionally, reefs can provide a food source for nekton (Harding \& Mann 2001, Grabowski 2004), leading to increased growth (Shervette \& Gelwick 2008) and subsequent survivorship 
(Sogard 1997, Stunz et al. 2002b), and these functional relationships should be further investigated. Overall, given the density patterns observed, large areal coverage of OYS in many estuaries and the evidence for these functional processes, all suggest that OYS may function as an important nursery area and essential fish habitat (Beck et al. 2001).

Acknowledgements. This research was conducted through the NOAA Fisheries Service Southeast Fisheries Science Center by personnel from the Fishery Ecology Branch (FEB) located at the Galveston Laboratory and the Harte Research Institute (HRI) for Gulf of Mexico Studies at Texas A\&M University-Corpus Christi. The assistance of everyone in the FEB and HRI was essential for the successful completion of this project. In particular, we thank Jim Ditty, Jennifer Doerr, Shawn Hillen, Kirk Kilfoyle, Juan Salas, Suraida NanezJames, Mike Lowe, Janelle Case, Harley Clinton, Jamie Eckles, Katherine Hess, Kelly Finn, Larry Perez, and Torrie Macbeth for logistical support, field collections, and laboratory sorting. Special thanks to Megan Reese Robillard and John Froeschke for critical evaluation of the manuscript and consultation on statistical analyses and Jason Williams for help creating Fig. 1. We acknowledge the NOAA Restoration Center, Southeast Fisheries Science Center, and the Texas Research Development Fund for funding this research project. The findings and conclusions in this paper are those of the authors and do not necessarily represent the views of the NOAA Fisheries Service. Finally, we would like to acknowledge 4 anonymous reviewers who provided insightful comments and suggestions that greatly improved this manuscript.

\section{LITERATURE CITED}

Baltz DM, Rakocinski C, Fleeger JW (1993) Microhabitat use by marsh-edge fishes in a Louisiana estuary. Environ Biol Fish 36:109-126

Beck MW, Heck KL, Able KW, Childers DL and others (2001) The identification, conservation, and management of estuarine and marine nurseries for fish and invertebrates. Bioscience 51:633-641

Blackmon DC, Eggleston DB (2001) Factors influencing planktonic, post-settlement dispersal of early juvenile blue crabs (Callinectes sapidus Rathbun). J Exp Mar Biol Ecol 257:183-203

Boesch DF, Turner RE (1984) Dependence of fishery species on salt marshes: the role of food and refuge. Estuaries 7: 460-468

Breitburg DL, Rose KA, Cowan JH (1999) Linking water quality to larval survival: predation mortality of fish larvae in an oxygen-stratified water column. Mar Ecol Prog Ser 178: $39-54$

Coen L, Grizzle R (2007) The importance of habitat created by molluscan shellfish to managed species along the Atlantic Coast of the United States. In: Thomas J, Nygard J (eds) Atlantic States Marine Fisheries Commission. Habitat Manag Ser 8:1-108

Coen LD, Luckenbach MW, Breitburg DL (1999) The role of oyster reefs as essential fish habitat: a review of current knowledge and some new perspectives. Am Fish Soc Symp 22:438-454

Coen LD, Brumbaugh RD, Bushek D, Grizzle R and others (2007) Ecosystem services related to oyster restoration. Mar Ecol Prog Ser 341:303-307
Day RW, Quinn GP (1989) Comparisons of treatments after an analysis of variance in ecology. Ecol Monogr 59:433-463

Day JW, Hall CAS, Kemp WM, Yanez-Arancibia A (1989) Estuarine ecology, Vol 1. John Wiley \& Sons, New York, NY

Eggleston DB, Etherington LL, Elis WE (1998) Organism response to habitat patchiness: species and habitatdependent recruitment of decapod crustaceans. J Exp Mar Biol Ecol 223:111-132

Gain I (2009) Oysters as nekton habitat in estuarine ecosystems. MS thesis, Texas A\&M University-Corpus Christi, Corpus Christi, TX

> Glancy TP, Frazer TK, Cichra CE, Lindberg WJ (2003) Comparative patterns of occupancy by decapod crustaceans in seagrass, oyster, and marsh-edge habitats in a northeast Gulf of Mexico estuary. Estuaries 26:1291-1301

Grabowski JH (2004) Habitat complexity disrupts predatorprey interactions but not the trophic cascade on oyster reefs. Ecology 85:995-1004

Grabowski JH, Hughes AR, Kimbro DL, Dolan MA (2005) How habitat setting influences restored oyster reef communities. Ecology 86:1926-1935

Grabowski JH, Hughes AR, Kimbro DL, Dolan MA (2008) Habitat complexity influences cascading effects of multiple predators. Ecology 89:3413-3422

Harding JM, Mann R (1999) Fish species richness in relation to restored oyster reefs, Piankatank River, Virginia. Bull Mar Sci 65:289-299

Harding JM, Mann R (2001) Oyster reefs as fish habitat: opportunistic use of restored reefs by transient fishes. J Shellfish Res 20:951-959

> Heck KL Jr, Thoman TA (1981) Experiments on predatorprey interactions in vegetated aquatic habitats. J Exp Mar Biol Ecol 53:125-134

Holt SA, Kitting CL, Arnold CR (1983) Distribution of young red drum among different sea-grass meadows. Trans Am Fish Soc 112:267-271

Irlandi EA, Crawford MK (1997) Habitat linkages: the effect of intertidal saltmarshes and adjacent subtidal habitats on abundance, movement, and growth of an estuarine fish. Oecologia 110:222-230

Kneib RT (1984) Patterns of invertebrate distribution and abundance in the intertidal salt-marsh - causes and questions. Estuaries 7:392-412

Lehnert RL, Allen DM (2002) Nekton use of subtidal oyster shell habitat in a southeastern U.S. estuary. Estuaries 25: 1015-1024

Lenihan HS, Grabowski JH, Thayer GW (1998) Recruitment to and utilization of oyster reef habitat by commercially and recreationally valuable crabs and fishes: an experiment with economic analysis. Final Report No. 1-97, National Marine Fisheries Service, Beaufort, NC

Lenihan HS, Peterson CH, Byers JE, Grabowski JH, Thayer GW, Colby DR (2001) Cascading of habitat degradation: oyster reefs invaded by refugee fishes escaping stress. Ecol Appl 11:764-782

> Levin P, Petrik R, Malone J (1997) Interactive effects of habitat selection, food supply and predation on recruitment of an estuarine fish. Oecologia 112:55-63

Mann R, Harding JM (1997) Trophic studies on constructed 'restored' oyster reefs. Report No. Grant/Contract No. CB993267-02-1, Chesapeake Bay Program Office, Annapolis, MD

Mann R, Harding JM (1998) Continuing trophic studies on constructed 'restored' oyster reefs. Report No. Grant/Contract No. CB993267-03, Chesapeake Bay Program Office, Annapolis, MD 
McNatt RA, Rice JA (2004) Hypoxia-induced growth rate reduction in two juvenile estuary-dependent fishes. J Exp Mar Biol Ecol 311:147-156

Meyer DL, Townsend EC (2000) Faunal utilization of created intertidal eastern oyster (Crassostrea virginica) reefs in the southeastern United States. Estuaries 23:34-45

- Micheli F, Peterson CH (1999) Estuarine vegetated habitats as corridors for predator movements. Conserv Biol 13:869-881

Minello TJ (1999) Nekton densities in shallow estuarine habitats of Texas and Louisiana and the identification of essential fish habitat. In: Benaka LR (ed) Fish habitat: essential fish habitat, and rehabilitation. Am Fish Soc Symp 22:438-454

Minello TJ, Webb JW (1997) Use of natural and created Spartina alterniflora salt marshes by fishery species and other aquatic fauna in Galveston Bay, Texas, USA. Mar Ecol Prog Ser 151:165-179

Minello TJ, Zimmerman RJ (1992) Utilization of natural and transplanted Texas salt marshes by fish and decapod crustaceans. Mar Ecol Prog Ser 90:273-285

Minello TJ, Able KW, Weinstein MP, Hays CG (2003) Salt marshes as nurseries for nekton: testing hypotheses on density, growth and survival through meta-analysis. Mar Ecol Prog Ser 246:39-59

Orth RJ, Heck KL Jr., van Montfrans J (1984) Faunal communities in seagrass beds: a review of the influence of plant structure and prey characteristics on predator-prey relationships. Estuaries 7:339-350

Pattillo ME, Czapla TE, Nelson DM, Monaco ME (1997) Distribution and abundance of fishes and invertebrates in Gulf of Mexico estuaries, Vol 2. Species life history summaries. Estuarine Living Marine Resources Program Report No. 11, National Oceanic and Atmospheric Administration/National Ocean Service Strategic Environmental Assessments Division, Silver Spring, MD

Perez Farfante I, Kensley B (1989) Penaeoid and sergestoid shrimps and prawns of the world: keys and diagnoses for the families and genera. Memoires du Museum National d'Histoire Naturelle, Tome 175, Paris

$>$ Peterson CH, Grabowski JH, Powers SP (2003) Estimated enhancement of fish production resulting from restoring oyster reef habitat: quantitative valuation. Mar Ecol Prog Ser 264:249-264

Pile AJ, Lipcius RN, van Montfrans J, Orth RJ (1996) Densitydependent settler-recruit-juvenile relationships in blue crabs. Ecol Monogr 66:277-300

Plunket J, La Peyre MK (2005) Oyster beds as fish and macroinvertebrate habitat in Barataria Bay, Louisiana. Bull Mar Sci 77:155-164

Pollard DA (1984) A review of ecological-studies on seagrass fish communities, with particular reference to recent studies in Australia. Aquat Bot 18:3-42

Posey MH, Alphin TD, Powell CM, Townsend E (1999) Oyster reefs as habitat for epibenthic fish and decapods. In: Luckenbach MW, Mann R, Wesson J (eds) Oyster reef habitat restoration: a synopsis of approaches. Virginia Institute of Marine Science Press, Williamsburg, VA, p 229-237

Powell EN, Song JG, Ellis MS, Wilson Ormond EA (1995) The status and long-term trends of oyster reefs in Galveston Bay, Texas. J Shellfish Res 14:439-457

Rice WR (1989) Analyzing tables of statistical tests. Evolution 43:223-225

Robblee MB, Zieman JC (1984) Diel variation in the fish fauna of a tropical seagrass feeding ground. Bull Mar Sci 34:335-345

Rodney WS, Paynter KT (2006) Comparisons of macrofaunal assemblages on restored and non-restored oyster reefs in mesohaline regions of Chesapeake Bay in Maryland. J Exp Mar Biol Ecol 335:39-51
Rozas LP (1995) Hydroperiod and its influence on nekton use of the salt marsh: a pulsing ecosystem. Estuaries 18: 579-590

Rozas LP, Minello TJ (1997) Estimating densities of small fishes and decapod crustaceans in shallow estuarine habitats: a review of sampling design with focus on gear selection. Estuaries 20:199-213

Rozas LP, Minello TJ (1998) Nekton use of salt marsh, seagrass, and nonvegetated habitats in a south Texas (USA) estuary. Bull Mar Sci 63:481-501

> Rozas LP, Minello TJ (2006) Nekton use of Vallisneria americana Michx. (wild celery) beds and adjacent habitats in coastal Louisiana. Estuaries Coasts 29:297-310

- Rozas LP, Odum WE (1988) The role of submerged aquatic vegetation in influencing the abundance of nekton on contiguous tidal fresh-water marshes. J Exp Mar Biol Ecol 114:289-300

$>$ Rozas LP, Zimmerman RJ (2000) Small-scale patterns of nekton use among marsh and adjacent shallow nonvegetated areas of the Galveston Bay Estuary, Texas (USA). Mar Ecol Prog Ser 193:217-239

Runyan S (1961) Early development of the clingfish, Gobiesox strumosus Cope. Chesap Sci 2:113-141

Shervette VR, Gelwick F (2007) Habitat-specific growth in juvenile pinfish. Trans Am Fish Soc 136:445-451

Shervette VR, Gelwick F (2008) Relative nursery function of oyster, vegetated marsh edge, and nonvegetated bottom habitats for juvenile white shrimp Litopenaeus setiferus. Wetlands Ecol Manag 16:405-419

Sogard SM (1997) Size-selective mortality in the juvenile stage of teleost fishes: a review. Bull Mar Sci 60: 1129-1157

Steimle FW, Zetlin C (2000) Reef habitats in the Middle Atlantic Bight: abundance, distribution, associated biological communities, and fishery resource use. Mar Fish Rev 62: $24-42$

Stunz GW, Levin PS, Minello TJ (2001) Selection of estuarine nursery habitats by wild-caught and hatchery-reared juvenile red drum in laboratory mesocosms. Environ Biol Fishes 61:305-313

Stunz GW, Minello TJ, Levin PS (2002a) A comparison of early juvenile red drum densities among various habitat types in Galveston Bay, Texas. Estuaries 25:76-85

Stunz GW, Minello TJ, Levin PS (2002b) Growth of newly settled red drum Sciaenops ocellatus in different estuarine habitat types. Mar Ecol Prog Ser 238:227-236

Szedlmayer ST, Howe JC (1997) Substrate preference in age-0 red snapper, Lutjanus campechanus. Environ Biol Fishes 50:203-207

Tolley SG, Volety AK (2005) The role of oysters in habitat use of oyster reefs by resident fishes and decapod crustaceans. J Shellfish Res 24:1007-1012

- Wannamaker CM, Rice JA (2000) Effects of hypoxia on movements and behavior of selected estuarine organisms from the southeastern United States. J Exp Mar Biol Ecol 249: 145-163

Weinstein MP (1979) Shallow marsh habitats as primary nurseries for fishes and shellfish, Cape Fear River, North Carolina. Fish Bull 77:339-357

Wenner E, Beatty HR, Coen L (1996) A method for quantitatively sampling nekton on intertidal oyster reefs. J Shellfish Res 15:769-775

Zimmerman R, Minello T, Zamora G (1984) Selection of vegetated habitat by brown shrimp, Penaeus aztecus, in Galveston Bay salt marsh. Fish Bull 82:325-336

Zimmerman R, Minello T, Baumer T, Castiglione M (1989) Oyster reef as habitat for estuarine macrofauna. NOAA Technical Memo. NMFS-SEFC 249:16

Submitted: September 28, 2009; Accepted: February 24, 2010

Proofs received from author(s): May 1, 2010 